\title{
INDUCTION OF SYSTEMIC OXIDATIVE STRESS LEADS TO BRAIN OEDEMA IN PORTACAVAL SHUNTED RATS
}

\author{
Cristina R. Bosoi, Mélanie Tremblay and Christopher F. Rose*
}

1. Neuroscience Research Unit, Hôpital Saint-Luc (CRCHUM), Université de Montréal, Québec, Canada

\section{* Correspondence}

Christopher F. Rose, PhD, Neuroscience Research Unit, Hôpital Saint-Luc (CRCHUM), Université de Montréal, Québec, Canada

Tel: +15148908000

Fax: +1 5144127253

\section{ABSTRACT}

Background \& Aims The pathogenesis of hepatic encephalopathy (HE) is multifactorial and often associated with the development of brain oedema. In addition to ammonia playing a central role, systemic oxidative stress is believed to aggravate the neuropsychological effects of ammonia in patients with chronic liver disease (CLD). The aim of this study was to (i) induce systemic oxidative stress in hyperammonaemic portacaval anastomosed (PCA) rats by inhibiting the antioxidant glutathione using Dimethyl maleate (DEM) and (ii) investigate whether a synergistic relationship between ammonia and oxidative stress contributes to the pathogenesis of brain oedema in CLD. Methods Four-week PCA and sham-operated rats received DEM $(0.4-4 \mathrm{mg} / \mathrm{kg} / \mathrm{day})$ for the last 10 days before sacrifice when oxidative stress markers [reactive oxygen species (ROS) and malondialdehyde (MDA)] were assessed in blood and frontal cortex. Brain water content was measured using a specific gravimetric technique. Results Dimethyl maleate induced an increase in ROS and MDA in the blood, but not in the brain, of the PCA rats, compared with non-treated PCA rats. This was accompanied with an increase in brain water content (PCA+DEM: $78.45 \pm 0.13 \%$ vs. PCA: $77.38 \pm 0.11 \%, P<0.001$ ). Higher doses of DEM induced systemic oxidative stress in sham-operated controls, but brain oedema did not develop. Conclusions Dimethyl maleate provoked systemic, not central, oxidative stress in PCA rats, resulting in the development of brain oedema. Independently, hyperammonaemia and systemic oxidative stress do not precipitate brain oedema; therefore, our findings sustain that a synergistic effect between hyperammonaemia and systemic oxidative stress is responsible for the development of brain oedema in HE.

Keywords:

hepatic encephalopathy;oxidative stress;brain oedema;portacaval shunt;dimethyl maleate

\section{Abbreviations}

CLD chronic liver disease; DEM dimethyl maleate; GSH glutathione; HE hepatic encephalopathy; PCA portacaval anastomosis; ROS reactive oxygen species

\section{INTRODUCTION}

Hepatic encephalopathy (HE) is a debilitating condition affecting close to 30-84\% of patients with chronic liver disease (CLD). This neuropsychiatric disorder is characterized by cognitive, psychiatric and motor disturbances and is primarily divided into overt (confusion, disorientation, ataxia and coma) and covert (reduced psychomotor speed, increased reaction

Bosoi, C.R., Tremblay, M. \& Rose, C.F., 2014. Induction of systemic oxidative stress leads to brain oedema in portacaval shunted rats. Liver International, 34(9), pp.1322-1329. 
This is a post-peer-review, pre-copyedit version of an article published in Liver International. The final authenticated version is available online at http://dx.doi.org/10.1111/liv.12414

time, sensory abnormalities, poor concentration) [1]. Contrary to overt HE, the diagnosis of covert HE is not clinically evident and requires sensitive psychometric and neurophysiological testing [2]. The burden of covert $\mathrm{HE}$ is multidimensional, affecting the patient's quality of life (ability to work and drive a car) and economically draining on health care systems [3]. More importantly, covert HE places patients at a higher risk of developing overt HE with increased mortality [4].

The neuropathology of HE in CLD reveals morphological changes in astrocytes, including cell swelling, which consequently leads to brain oedema [5]. This feature is commonly observed in both patients and rats with cirrhosis suffering from covert HE [6-11], however, $\mathrm{HE}$ also exists in the absence of brain oedema [12, 13]. Brain oedema has demonstrated to correlate with the severity of the disease $[15,14,16]$, and has shown to improve following liver transplantation [6].

Liver failure induces hyperammonaemia, which consequently leads to neurotoxic levels of ammonia, a principle factor long considered to be implicated in the pathogenesis of HE. However, a correlation between levels of blood ammonia and severity of HE in CLD remains controversial [17-21], suggesting other pathogenic factors are involved. Recently, it has been postulated that systemic oxidative stress can exacerbate the neurological effects of ammonia and play an important role in the pathogenesis of HE in CLD [22, 23]. Oxidative stress, a systemic phenomenon correlated with severity of liver disease [24, 25], is an important condition present in patients with cirrhosis, and occurs due to an imbalance between the activities of pro-oxidants and antioxidants, leading to a surplus in reactive oxygen species (ROS). Pro-oxidants constitutively generate ROS, which are important for proper cell signalling and function whereas antioxidants are responsible for regulating the levels of ROS and thus maintain a healthy equilibrium. Disturbance in pro-oxidant/antioxidant harmony has been demonstrated in the setting of liver disease. Xanthine oxidase, a liver derived pro-oxidant enzyme which produces ROS following oxidation of hypoxanthine to xanthine and to uric acid, has been found to be increased in circulation in both patients with CLD and cirrhotic rats [26, 27]. In addition, we recently demonstrated that by inhibiting xanthine oxidase with allopurinol, circulating levels of ROS were attenuated, brain oedema was prevented and HE was improved [27]. Furthermore, glutathione (GSH), an important antioxidant synthesized by the liver, has been found to be decreased in cirrhosis [27-29].

Contrary with cirrhotic rats, rats with portacaval anastomosis (PCA) develop hyperammonaemia in the absence of intrinsic hepatocellular disease [30] and neither brain oedema nor any evidence of oxidative stress has been observed [27, 31]. The aim of this study was to induce systemic oxidative stress in PCA rats and investigate whether a synergistic relationship between ammonia and oxidative stress is key in the pathogenesis of brain oedema in CLD. To induce oxidative stress, PCA rats were treated with dimethyl maleate (DEM), an irreversible GSH inhibitor [32].

\section{MATERIALS AND METHODS}

\section{Animal model and experimental protocol}

Adult male Sprague-Dawley rats weighing 200-225 g were anesthetized with isoflurane, and an end-to-side PCA was performed [27]. Starting at day 18 after surgery, both PCA and sham-operated controls were administered DEM once daily $(4,3,2,1$ or $0.4 \mathrm{mg} / \mathrm{kg}$ diluted in $1 \mathrm{ml} / \mathrm{kg}$ saline; intraperitoneally) until day 28 when they were sacrificed. Vehicle-treated sham and PCA rats received equivalent volumes of saline. Experiments were conducted following the Guidelines of Canadian Council on Animal Care and were approved by the Animal Protection Committee of CRCHUM.

\section{Markers of oxidative stress}

Circulating ROS were evaluated following the oxidation of dichlorofluorescein diacetate (Invitrogen, Carlsbad, CA, USA) to dichlorofluorescein as previously described [27]. Plasmatic and/or frontal cortex lipid peroxidation was estimated by measuring the levels of malondialdehyde (MDA) and 4-hydroxy-2-nonenal (HNE) formation. MDA levels were measured using TBARS Assay Kit (Cayman Chemical Company, Ann Arbor, MI, USA), according to the manufacturer's protocol.

Total GSH was measured in the plasma and frontal cortex tissue, based on the sulphhydryl group oxidation by DTNB $\left(5,5^{\prime}-\right.$ dithiobis-2-nitrobenzoic acid), which forms a yellow product (5-thio-2-nitrobenzoic acid, TNB) that is measured

Bosoi, C.R., Tremblay, M. \& Rose, C.F., 2014. Induction of systemic oxidative stress leads to brain oedema in portacaval shunted rats. Liver International, 34(9), pp.1322-1329. 
This is a post-peer-review, pre-copyedit version of an article published in Liver International. The final authenticated version is available online at http://dx.doi.org/10.1111/liv.12414

spectrophotometrically. Oxidized GSH was previously reduced to GSH by glutathione reductase. Plasma and brain samples were extracted in five volumes of sulphosalicylic acid 5\%, then homogenized and centrifuged $10 \mathrm{~min}$ at $13000 \mathrm{~g} .10 \mu \mathrm{l}$ of the supernatant was incubated for $5 \mathrm{~min}$ with $150 \mu \mathrm{l}$ sodium phosphate-buffer $95 \mathrm{mM}$ containing EDTA $0.95 \mathrm{mM}$, glutathione reductase $0.115 \mathrm{U} / \mathrm{ml}$ and DTNB $0.031 \mathrm{mg} / \mathrm{ml}$. The reaction was started by adding $50 \mu 1$ reduced nicotinamide adenine dinucleotide phosphate (NADPH) $0.038 \mathrm{mg} / \mathrm{ml}$, the oxidation of which was followed at $412 \mathrm{~nm}$ for $32 \mathrm{~min}$. Total GSH concentration was calculated based on a standard curve with known concentrations of GSH.

For HNE assessment, frontal cortex was homogenized in lysis buffer (50 mM Tris pH 7.5, 1 mM EDTA, 1/500 cold protease inhibitor cocktail). Homogenates were centrifuged $40 \mathrm{~min}$ at $13000 \mathrm{~g}$ at $4^{\circ} \mathrm{C}$. The supernatant was taken and protein content was determined according to the method of Lowry et al. [33]. Samples containing $75 \mu \mathrm{g}$ of cortex proteins were separated by $8 \%$ sodium dodecyl sulphate-polyacrylamide gels electrophoresis, and then transferred to polyvinylidene difluoride membranes. Membranes were blocked with 5\% skim milk in TBS-T buffer $(1 \mathrm{mM}$ Tris $\mathrm{pH}=7.5,10 \mathrm{mM} \mathrm{NaCl}$ and $0.5 \%$ Tween 20 ) for $1 \mathrm{~h}$ at room temperature and followed by $1 \mathrm{~h}$ incubation with rabbit polyclonal anti-HNE antibody (Calbiochem, Darmstadt Germany) at a dilution of 1:1000. Membranes were washed three times in TBS-T buffer for 5 min and then incubated for $1 \mathrm{~h}$ at room temperature, with the corresponding secondary antibody labelled with horseradish peroxidase (Perkin-Elmer, Waltham, MA, USA) at a dilution of 1:10 000 and washed three times in TBS-T buffer for 5 min. Immunoreactivity was detected with chemiluminescence reagent and probed on X-ray film. A monoclonal antibody to $\beta$-actin (Sigma, St-Louis, MO, USA) was used at a dilution of 1:200 000 as a control of protein loading.

\section{Brain water content}

Brain water content was measured using the sensitive densitometry technique. Frontal cortex was freshly dissected at $4^{\circ} \mathrm{C}$ and cut into $2 \mathrm{~mm}^{3}$ pieces. Tissue pieces were placed in density gradient columns and equilibrium point was recorded after 2 min. Columns were made with different kerosene and bromobenzene mixtures and precalibrated with $\mathrm{K}_{2} \mathrm{SO}_{4}$ solutions of known densities. At least eight samples were measured in each rat. Water content was calculated based on tissue density, according to the formula described by Marmarou et al. [34].

\section{Liver biochemistry}

Liver necrosis markers aspartate and alanine aminotransferase were measured using routine clinical biochemistry techniques.

\section{Ammonia}

Plasmatic ammonia levels were measured using a commercial kit (Sigma) according to the manufacturer's protocol. The kit is based on the reaction of ammonia with $\alpha$-ketoglutarate and reduced nicotinamide adenine dinucleotidephosphate in the presence of L-glutamate dehydrogenase. Oxidation rate of reduced NADPH was recorded by the absorbance decrease at $340 \mathrm{~nm}$. Ammonia concentration was calculated according to the manufacturer's protocol.

\section{Statistical analysis}

Data are expressed as mean \pm standard error of the mean. Significance of difference was tested with Anova, followed by Newman-Keuls post-test using GraphPad Prism4 (La Jolla, CA, USA). Probability values $P<0.05$ were considered to be statistically significant.

\section{RESULTS}

\section{Dimethyl maleate treatment}

It has previously been demonstrated that a single injection of DEM at doses up to $4 \mathrm{mg} / \mathrm{kg}$ administered to naïve rats was non-toxic and did not lead to mortality [32]. In our study, since oxidative stress is a chronic feature observed throughout the time course of cirrhosis [27], we aimed to induce long-term oxidative stress in PCA rats by injecting DEM daily for 10 days at different doses $(4,3,2,1$ or $0.4 \mathrm{mg} / \mathrm{kg})$. DEM at doses 4 and $3 \mathrm{mg} / \mathrm{kg}$ lead to $100 \%$ mortality in PCA rats before the end of the 10-day treatment (4 mg/kg resulted in death after 1-2 days of treatment, and $3 \mathrm{mg} / \mathrm{kg}$ resulted in death following 7-

Bosoi, C.R., Tremblay, M. \& Rose, C.F., 2014. Induction of systemic oxidative stress leads to brain oedema in portacaval shunted rats. Liver International, 34(9), pp.1322-1329. 
9 days of treatment), whereas DEM at doses 2,1 and $0.4 \mathrm{mg} / \mathrm{kg}$ did not lead to mortality in PCA rats. No mortality occurred in sham-operated controls treated with DEM at doses $4,3,2,1$ and $0.4 \mathrm{mg} / \mathrm{kg}$ for the duration of the 10-day treatment.

\section{Dimethyl maleate induces systemic oxidative stress in portacaval anastomosis rats}

The efficacy of DEM treatment in inducing systemic ROS was assessed by measuring various markers of oxidative stress. A significant increase in plasma ROS was observed in PCA rats vs. sham-operated controls following DEM treatment at doses 2 and $1 \mathrm{mg} / \mathrm{kg}$, but not at $0.4 \mathrm{mg} / \mathrm{kg}$ (Fig. 1A). Continuing with the dose $1 \mathrm{mg} / \mathrm{kg}$, DEM treatment led to a significant decrease in total GSH in PCA rats, but not in sham-operated controls compared with respective non-treated controls (Fig. 1B). MDA, a reliable marker of oxidative stress resulting from lipid peroxidation, was found to be significantly increased in DEM-treated PCA rats compared with sham-operated controls (Fig. 1C).
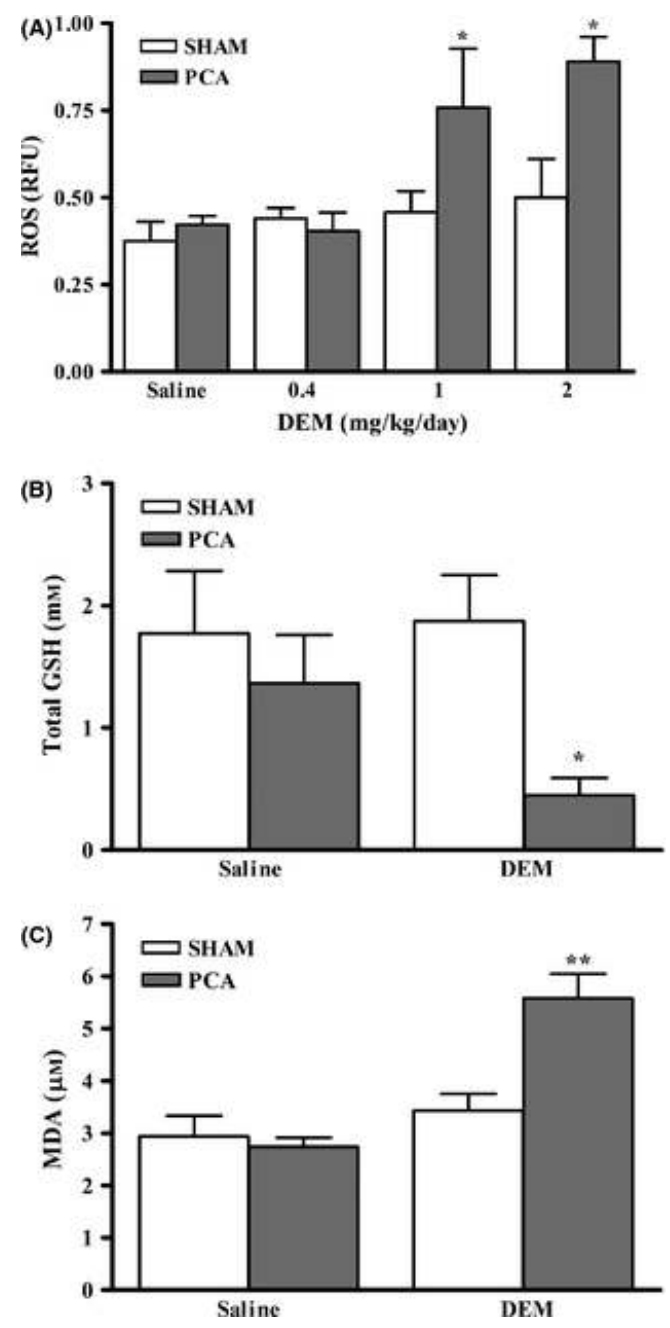

Figure 1. (A) Circulating reactive oxygen species (ROS) in rats with portacaval anastomosis (PCA) and sham-operated controls treated and not treated with dimethyl maleate (DEM) at a dose of 2, 1 or $0.4 \mathrm{mg} / \mathrm{kg} /$ day for 10 days; (B) plasmatic glutathione (GSH) and (C) malondialdehyde (MDA) in rats with PCA and sham-operated controls treated and not treated with DEM at a dose of $1 \mathrm{mg} / \mathrm{kg} /$ day for 10 days. Data are expressed as mean \pm SEM. $* P<0.05, * * P<0.01$, significantly different from all other groups.

\section{Dimethyl maleate induces brain oedema in portacaval anastomosis rats}

Bosoi, C.R., Tremblay, M. \& Rose, C.F., 2014. Induction of systemic oxidative stress leads to brain oedema in portacaval shunted rats. Liver International, 34(9), pp.1322-1329. 
Dimethyl maleate treatment to PCA rats lead to a significant increase in brain water content in frontal cortex compared with non-treated PCA rats (PCA + DEM: $78.06 \pm 0.09 \%$ vs. non-treated PCA: $77.53 \pm 0.12 \%, P<0.05)$ and compared with sham-operated controls $(77.54 \pm 0.11 \%, P<0.05)$ (Fig. 2$)$.

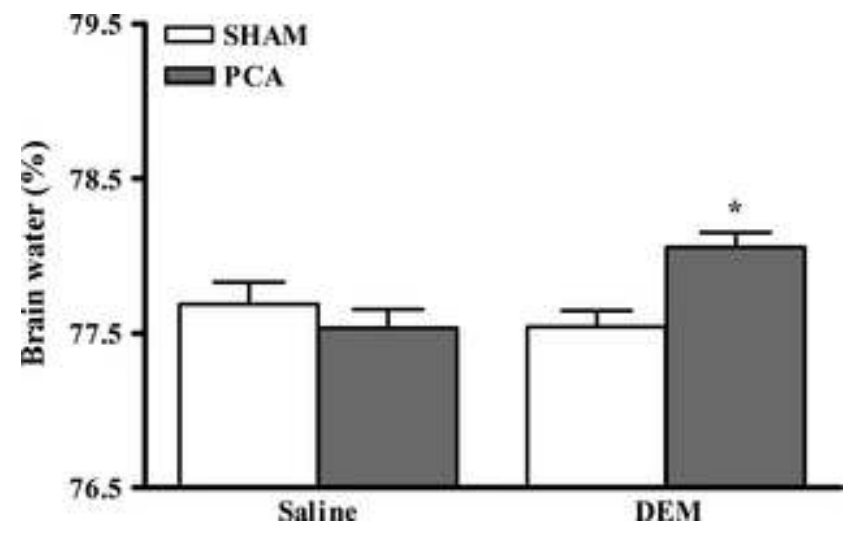

Figure 2. Frontal cortex water content in rats with portacaval anastomosis (PCA) and sham-operated controls treated and not treated with dimethyl maleate (DEM) at a dose of $1 \mathrm{mg} / \mathrm{kg} /$ day for 10 days. Data are expressed as mean \pm SEM. $* P<0.05$, significantly different from all other groups.

\section{Dimethyl maleate does not induce cerebral oxidative stress in portacaval anastomosis rats}

Dimethyl maleate treatment did not lead to a significant increase in oxidative stress markers (total GSH, MDA and HNE) in either the frontal cortex of PCA nor sham-operated controls (Fig. 3A-C).
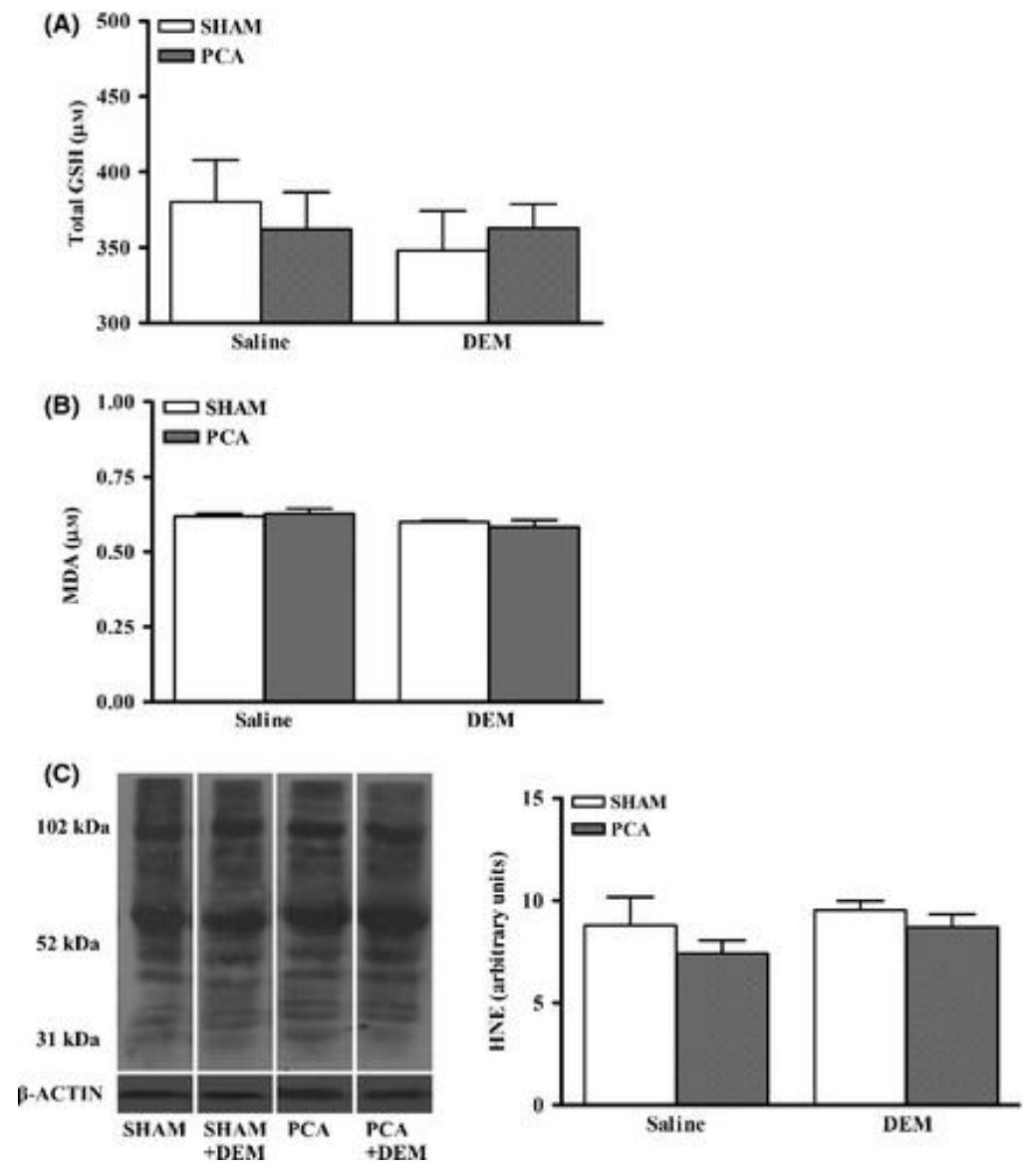

Bosoi, C.R., Tremblay, M. \& Rose, C.F., 2014. Induction of systemic oxidative stress leads to brain oedema in portacaval shunted rats. Liver International, 34(9), pp.1322-1329. 
Figure 3. (A) Cerebral glutathione (GSH); (B) malondialdehyde (MDA) and (C) 4-hydroxy-2- nonenal (HNE; representative immunoblotting images for each group along with densitometric quantification are shown) in rats with portacaval anastomosis (PCA) and sham-operated controls treated and not treated with dimethyl maleate (DEM) at a dose of $1 \mathrm{mg} / \mathrm{kg} /$ day for 10 days. Data are expressed as mean \pm SEM.

\section{Dimethyl maleate does not affect ammonia levels and liver function}

Dimethyl maleate treatment did not induce hyperammonaemia in sham-operated controls, nor influence the blood ammonia levels in PCA rats. In addition, DEM treatment did not increase the levels of AST or ALT in both PCA and sham-operated controls (Table 1).

Table 1. Hepatic function markers aspartate aminotransferase (AST) and alanine aminotransferase (ALT) and ammonia levels in rats with portacaval anastomosis (PCA) and sham-operated controls treated and not treated with dimethyl maleate (DEM) at a dose of $1 \mathrm{mg} / \mathrm{kg} / \mathrm{day}$ for 10 days

\begin{tabular}{llccc}
\hline & Sham & PCA & Sham + DEM & PCA + DEM \\
\hline AST (U/L) & $73.00 \pm 5.02$ & $85.50 \pm 3.01$ & $66.00 \pm 1.03$ & $80.00 \pm 6.69$ \\
ALT $(\mathrm{U} / \mathrm{L})$ & $45.60 \pm 3.26$ & $48.20 \pm 2.43$ & $41.00 \pm 5.00$ & $49.75 \pm 6.39$ \\
Ammonia $(\mu \mathrm{M})$ & $44.04 \pm 8.81$ & $189.01 \pm 17.84^{\star \star \star *}$ & $64.07 \pm 9.56$ & $152.10 \pm 27.15^{\star \star \star}$ \\
\hline
\end{tabular}

Data are expressed as mean \pm SEM. ${ }^{a} P<0.001$, significantly vs. sham-operated rats.

\section{Systemic oxidative stress independently does not lead to brain oedema}

Contrary with doses $0.4,1,2$ and $3 \mathrm{mg} / \mathrm{kg}$, DEM at dose $4 \mathrm{mg} / \mathrm{kg}$ provoked a significant increase in plasma ROS in shamoperated controls, which is similar to the increase in levels of ROS as those seen in PCA rats treated with DEM at a dose of $1 \mathrm{mg} / \mathrm{kg}$. However, the former was not accompanied with the apparition of brain oedema, as was observed in the DEMtreated PCA rats (Fig. 4).

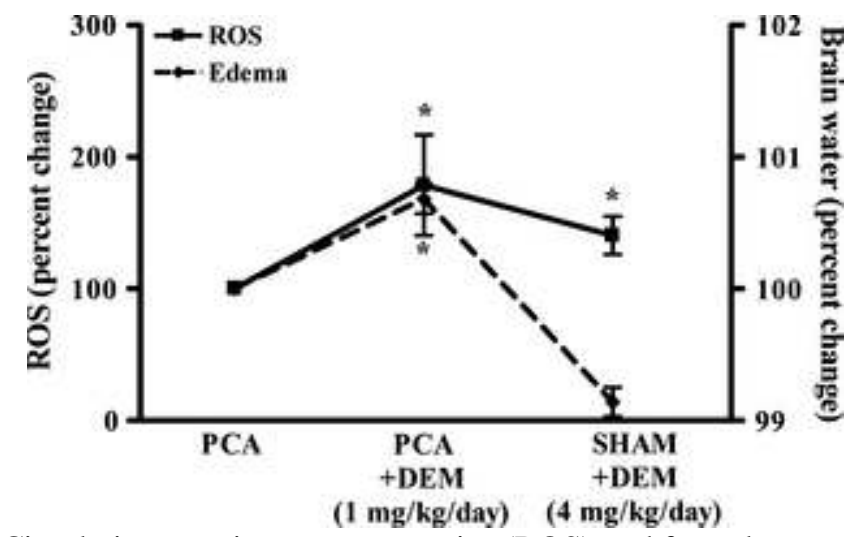

Figure 4. Circulating reactive oxygen species (ROS) and frontal cortex water in rats with portacaval anastomosis (PCA) treated and not treated with dimethyl maleate (DEM) at a dose of $1 \mathrm{mg} / \mathrm{kg} / \mathrm{day}$ for 10 days and sham-operated controls treated with dimethyl maleate (DEM) at a dose of $4 \mathrm{mg} / \mathrm{kg} /$ day for 10 days. Data are expressed as percent change compared with non-treated shamoperated controls. ROS percent change is represented on the left axis and traced with a full line, while brain oedema is represented on the right axis and traced with a dotted line. ${ }^{*} P<0.05$, significantly vs. non-treated PCA rats.

\section{DISCUSSION}

Bosoi, C.R., Tremblay, M. \& Rose, C.F., 2014. Induction of systemic oxidative stress leads to brain oedema in portacaval shunted rats. Liver International, 34(9), pp.1322-1329. 
This is a post-peer-review, pre-copyedit version of an article published in Liver International. The final authenticated version is available online at http://dx.doi.org/10.1111/liv.12414

Dimethyl maleate treatment significantly resulted in a reduction in circulating levels of GSH in hyperammonaemic PCA rats, which in turn led to the induction of systemic, but not central, oxidative stress, and triggered the apparition of brain oedema. DEM-induced systemic oxidative stress, in the absence of hyperammonaemia, was not accompanied with presence of brain oedema sustaining that both hyperammonaemia and systemic oxidative stress are synergistically implicated in the pathogenesis of brain oedema in CLD.

The aim of this study was to induce oxidative stress in hyperammonaemic PCA rats by targeting and diminishing the antioxidant GSH, a tripeptide formed of glutamate, cysteine and glycine, whose cysteine residue acts as an electron donor. By scavenging ROS, GSH is converted into oxidized glutathione and reduced back to GSH by glutathione reductase. GSH, primarily produced by the liver, has been found to be increased in early stages of cirrhosis [35] but is evidently diminished during late stages of liver disease [27-29]. However, in PCA rats, where the liver function is preserved, GSH levels are not affected [27]. To target the inhibition of GSH, we used DEM, a direct GSH inhibitor that acts by irreversibly conjugating the cysteine's sulphahydryl group. It has been previously demonstrated that a single dose of DEM $(600 \mathrm{mg} / \mathrm{kg})$ administered to naïve rats induces a transient (12 h) decrease in hepatic and cerebral GSH [36]. To simulate a chronic condition of oxidative stress (as observed during cirrhosis), daily injections of smaller doses of DEM were administered. Ten-day treatment of DEM, as low as $1 \mathrm{mg} / \mathrm{kg}$, significantly diminished GSH levels in PCA rats. This resulted in the induction of oxidative stress in plasma, reflected with an increase in ROS and MDA. This same dose of DEM did not lead to similar results in sham-operated treated rats. Instead, a higher dose of DEM $(4 \mathrm{mg} / \mathrm{kg})$ was needed to attain a similar significant increase in ROS. This suggests that PCA rats demonstrate a higher susceptibility to a DEM-induced oxidative stress insult compared with sham-operated controls. The mechanisms underlying this susceptibility are unresolved, but the effect of chronic moderate elevated levels of ammonia in PCA rats may render the brain sensitive to insults. The mechanisms underlying increased brain sensitivity following chronic ammonia exposure merits to be further investigated.

Ammonia has long been considered to play a major role in the pathogenesis of brain oedema and HE; however, its weak relationship with severity of $\mathrm{HE}$ suggests that other factors are involved. Our results suggest that systemic oxidative stress is a key factor, in addition to ammonia that precipitates brain oedema, since (i) hyperammonaemic PCA rats do not develop brain oedema and (ii) DEM-induced oxidative stress in sham-operated rats, which do not present hyperammonaemia, does not lead to brain oedema. This implies that neither oxidative stress nor ammonia independently induces brain oedema. PCA in the rat represents a type B HE model resulting from portal-systemic shunting in the absence of parenchymal liver disease.

Therefore, in the absence of liver-derived factors like inflammation and oxidative stress, brain oedema does not develop as previously demonstrated [12, 13, 27]. Since DEM-induced oxidative stress in hyperammonaemic PCA rats lead to the development of brain oedema, this implies that a synergistic effect between ammonia and oxidative stress is required to cause an increase in brain water.

These observations support our previous studies demonstrating that a synergistic effect between systemic oxidative stress and ammonia is imperative in the development of brain oedema in CLD [9, 27]. Furthermore, the clinical implications of this important synergy is sustained by a study from Montoliu et al. who elegantly demonstrated in cirrhotic patients with similar degrees of hypermamonaemia, that the detection of 3-nitrotyrosine in plasma distinguished covert HE cirrhotic patients from those without HE [37]. During liver disease, oxidative stress represents a systemic phenomenon [38], however, the pathophysiological significance of whether oxidative stress vs. ammonia is the 'first' or 'second' remains to be defined.

Swelling of the brain in DEM-treated PCA rats occurred in the absence of any indications of oxidative stress. It has been demonstrated that ammonia levels $>1 \mathrm{mM}$ lead to the generation of ROS in cultured astrocytes and cell swelling [39, 40]. Similarly, in vivo, acute ammonia toxicity also leads to oxidative stress in the brain by increasing ROS production and diminishing antioxidant enzymes activities [41, 42]. Nevertheless, ammonia concentrations $<500 \mu \mathrm{M}$ have been shown not to induce oxidative stress in both astrocyte cultures and brain slices [43, 44]. Furthermore, in both PCA rats and cirrhotic rats with brain ammonia levels $<250 \mu \mathrm{M}$, no signs of oxidative stress in the brain have been identified [27]. However, signs of nitrosative stress have been demonstrated in the brains of PCA rats [45] which suggests that nitrosative and oxidative stress may be implicated in different stages of $\mathrm{HE}$ and/or disease. Furthermore, there is strong evidence demonstrating that

Bosoi, C.R., Tremblay, M. \& Rose, C.F., 2014. Induction of systemic oxidative stress leads to brain oedema in portacaval shunted rats. Liver International, 34(9), pp.1322-1329. 
This is a post-peer-review, pre-copyedit version of an article published in Liver International. The final authenticated version is available online at http://dx.doi.org/10.1111/liv.12414

oxidative stress in the brain is associated with severe (overt) HE. Both acute ammonia intoxication and acute liver leading to severe HE and coma in rats [45-47], as well as cirrhotic patients who died with severe HE (grades III or IV) [48], markers of oxidative stress were detected in the brain. Overall, this suggests that the appearance of oxidative stress in the brain may be a vital stimulating factor in the pathogenesis of severe, overt HE.

The blood-brain barrier (BBB) is a structural barrier which impedes the influx of neurotoxic compounds from blood to brain. It has been previously demonstrated that circulating ROS can induce BBB oxidative damage and lead to tight junction protein modifications, resulting in BBB breakdown, as observed in stroke or sepsis [49]. However, in different animal models of covert HE, the BBB has been proven to be anatomically intact [11, 27]. This implies, in the setting of CLD, that ROS may not be causing structural breakdown to the BBB, but rather, through oxidative modifications of proteins and lipids, may stimulate changes in BBB signalling and permeability. These pathophysiological mechanisms merit to be investigated in relation systemic oxidative stress and the development of brain oedema in HE.

In the present study, we assessed oxidative stress and brain oedema in the frontal cortex; an important region for cognitive function. However, the heterogeneity of the brain does not insinuate all areas of the brain react similarly. In fact, the cerebellum has demonstrated to have an enhanced susceptibility to oxidative stress in hyperammonaemic rats [46]. Therefore, we do not exclude the possibility that other cerebral regions are affected.

During end stage liver disease, multiple factors other than ammonia and oxidative stress may also be contributing to the pathogenesis of brain oedema. Inflammation is an important factor, demonstrated to play an important role in inducing brain oedema [50] and known to play an important role in the cognitive and motor alterations in PCA rats [13, 51]. Although inflammation is believed to be tightly associated with oxidative stress, their relationship remains unresolved. Other factors such as alterations of energy metabolism, osmotic changes triggered by increases in glutamate, glutamine, lactate or expression in water or ion channels have also been demonstrated to play a role in brain oedema during liver disease, as reviewed by Bosoi and Rose [5]. Neither DEM-treated or untreated PCA rats demonstrated any affects in liver function, which excludes the roles of the above-mentioned factors, which have been found to be induced from the ailing liver during disease [52].

In conclusion, our study confirms that chronic hyperammonaemia and systemic (not central) oxidative stress independently do not lead to the development of brain oedema; however, when both factors are present, they act synergistically to induce an increase in brain water. The presence of brain oedema in covert HE may be a predisposing factor leading to a higher risk of triggering oxidative stress in the brain and developing overt HE. These results support that in addition to targeting ammonia producing/removing pathways, antioxidants or pro-oxidant inhibitors should be considered for the treatment of HE.

\section{ACKNOWLEDGEMENT}

Conflict of interest: The authors do not have any disclosures to report.

\section{REFERENCES}

1. Bajaj JS. Review article: the modern management of hepatic encephalopathy. Aliment Pharmacol Ther 2010; 31: 537-47.

2. Córdoba J. New assessment of hepatic encephalopathy. J Hepatol 2011; 54: 1030-40.

3. Bajaj JS, Wade JB, Sanyal AJ. Spectrum of neurocognitive impairment in cirrhosis: implications for the assessment of hepatic encephalopathy. Hepatology 2009; 50: 2014-21.

4. Hartmann IJ, Groeneweg M, Quero JC, et al. The prognostic significance of subclinical hepatic encephalopathy. Am J Gastroenterol 2000; 95: 2029-34.

5. Bosoi CR, Rose CF. Brain edema in acute liver failure and chronic liver disease: similarities and differences. Neurochem Int 2013; 62: 446-57.

6. Rovira A, Mínguez B, Aymerich FX, et al. Decreased white matter lesion volume and improved cognitive function after liver transplantation. Hepatology 2007; 46: 1485-90.

Bosoi, C.R., Tremblay, M. \& Rose, C.F., 2014. Induction of systemic oxidative stress leads to brain oedema in portacaval shunted rats. Liver International, 34(9), pp.1322-1329. 
This is a post-peer-review, pre-copyedit version of an article published in Liver International. The final authenticated version is available online at http://dx.doi.org/10.1111/liv.12414

7. Shah NJ, Neeb H, Kircheis G, et al. Quantitative cerebral water content mapping in hepatic encephalopathy. Neuroimage 2008; 41: 706-17.

8. Sugimoto R, Iwasa M, Maeda M, et al. Value of the apparent diffusion coefficient for quantification of low-grade hepatic encephalopathy. Am J Gastroenterol 2008; 103: 1413-20.

9. Bosoi CR, Parent-Robitaille C, Anderson K, Tremblay M, Rose CF. AST-120 (spherical carbon adsorbent) lowers ammonia levels and attenuates brain edema in bile duct-ligated rats. Hepatology 2011; 53: 1995-2002.

10. Davies NA, Wright G, Ytrebø LM, et al. L-ornithine and phenylacetate synergistically produce sustained reduction in ammonia and brain water in cirrhotic rats. Hepatology 2009; 50: 155-64.

11. Wright G, Davies NA, Shawcross DL, et al. Endotoxemia produces coma and brain swelling in bile duct ligated rats. Hepatology 2007; 45: 1517-26.

12. Erceg S, Monfort P, Hernández-Viadel M, et al. Oral administration of sildenafil restores learning ability in rats with hyperammonemia and with portacaval shunts. Hepatology 2005; 41: 299-306.

13. Cauli O, Rodrigo R, Piedrafita B, et al. Neuroinflammation contributes to hypokinesia in rats with hepatic encephalopathy: ibuprofen restores its motor activity. J Neurosci Res 2009; 87: 1369-74.

14. Lodi R, Tonon C, Stracciari A, et al. Diffusion MRI shows increased water apparent diffusion coefficient in the brains of cirrhotics. Neurology 2004; 62: 762-6.

15. Kale RA, Gupta RK, Saraswat VA, et al. Demonstration of interstitial cerebral edema with diffusion tensor MR imaging in type C hepatic encephalopathy. Hepatology 2006; 43: 698-706.

16. Zhang L, Qi R, Wu S, et al. Brain default-mode network abnormalities in hepatic encephalopathy: a resting-state functional MRI study. Hum Brain Mapp 2012; 33: 1384-92.

17. Ong JP, Aggarwal A, Krieger D, et al. Correlation between ammonia levels and the severity of hepatic encephalopathy. Am J Med 2003; 114: 188-93.

18. Kundra A, Jain A, Banga A, Bajaj G, Kar P. Evaluation of plasma ammonia levels in patients with acute liver failure and chronic liver disease and its correlation with the severity of hepatic encephalopathy and clinical features of raised intracranial tension. Clin Biochem 2005; 38: 696-9.

19. He Y, Li G, Song H, et al. Partial pressure of $\mathrm{NH}_{3}$ in cirrhotic patients with and without hepatic encephalopathy. J Gastrointest Liver Dis 2011; 20: 169-74.

20. Nicolao F, Efrati C, Masini A, et al. Role of determination of partial pressure of ammonia in cirrhotic patients with and without hepatic encephalopathy. J Hepatol 2003; 38: 441-6.

21. Weissenborn K, Ahl B, Fischer-Wasels D, et al. Correlations between magnetic resonance spectroscopy alterations and cerebral ammonia and glucose metabolism in cirrhotic patients with and without hepatic encephalopathy. Gut 2007; 56: $1736-42$.

22. Seyan AS, Hughes RD, Shawcross DL. Changing face of hepatic encephalopathy: role of inflammation and oxidative stress. World J Gastroenterol 2010; 16: 3347-57.

23. Bosoi CR, Rose CF. Oxidative stress: a systemic factor implicated in the pathogenesis of hepatic encephalopathy. Metab Brain Dis 2013; 28: 175-8.

24. Chen M-F, Mo L-R, Lin R-C, et al. Increase of resting levels of superoxide anion in the whole blood of patients with decompensated liver cirrhosis. Free Radic Biol Med 1997; 23: 672-9.

25. Spahr L, Bresson-Hadni S, Amann P, et al. Allopurinol, oxidative stress and intestinal permeability in patients with cirrhosis: an open-label pilot study. Liver Int 2007; 27: 54-60.

26. Battelli MG, Musiani S, Valgimigli M, et al. Serum xanthine oxidase in human liver disease. Am J Gastroenterol 2001; 96: 11949.

27. Bosoi CR, Yang X, Huynh J, et al. Systemic oxidative stress is implicated in the pathogenesis of brain edema in rats with chronic liver failure. Free Radic Biol Med 2012; 52: 1228-35.

28. Townsend DM, Tew KD, Tapiero H. The importance of glutathione in human disease. Biomed Pharmacother 2003; 57: 145-55.

29. Burgunder JM, Lauterburg BH. Decreased production of glutathione in patients with cirrhosis. Eur J Clin Invest 1987; 17: 40814.

30. Butterworth RF, Norenberg MD, Felipo V, et al. Experimental models of hepatic encephalopathy: ISHEN guidelines. Liver Int 2009; 29: 783-8.

31. Yang X, Bosoi CR, Jiang W, Tremblay M, Rose CF. Portacaval anastomosis-induced hyperammonemia does not lead to oxidative stress. Metab Brain Dis 2010; 25: 11-5.

32. Agarwal R, Shukla GS. Potential role of cerebral glutathione in the maintenance of blood-brain barrier integrity in rat. Neurochem Res 1999; 24: 1507-14.

33. Lowry OH, Rosebrough NJ, Farr AL, Randall RJ. Protein measurement with the Folin phenol reagent. J Biol Chem 1951; 193: 265-75.

Bosoi, C.R., Tremblay, M. \& Rose, C.F., 2014. Induction of systemic oxidative stress leads to brain oedema in portacaval shunted rats. Liver International, 34(9), pp.1322-1329. 
34. Marmarou A, Poll W, Shulman K, Bhagavan H. A simple gravimetric technique for measurement of cerebral edema. J Neurosurg 1978; 49: 530-7.

35. Ergün Y, Kurutaş EB, Ozdil B, Güneşaçar R, Ergün Y. Evaluation of nitrite/nitrate levels in relation to oxidative stress parameters in liver cirrhosis. Clin Res Hepatol Gastroenterol 2011; 35: 303-8.

36. Weber CA, Duncan CA, Lyons MJ, Jenkinson SG. Depletion of tissue glutathione with diethyl maleate enhances hyperbaric oxygen toxicity. Am J Physiol 1990; 258: L308-12.

37. Montoliu C, Cauli O, Urios A, et al. 3-nitro-tyrosine as a peripheral biomarker of minimal hepatic encephalopathy in patients with liver cirrhosis. Am J Gastroenterol 2011; 106: 1629-37.

38. Ljubuncic P, Tanne Z, Bomzon A. Evidence of a systemic phenomenon for oxidative stress in cholestatic liver disease. Gut 2000; 47: 710-6.

39. Murthy CR, Rama Rao KV, Bai G, Norenberg MD. Ammonia-induced production of free radicals in primary cultures of rat astrocytes. J Neurosci Res 2001; 66: 282-8.

40. Jayakumar AR, Panickar KS, Murthy CRK, Norenberg MD. Oxidative stress and mitogen-activated protein kinase phosphorylation mediate ammonia-induced cell swelling and glutamate uptake inhibition in cultured astrocytes. J Neurosci 2006; 26: 4774-84.

41. Kosenko E, Kaminsky Y, Kaminsky A, et al. Superoxide production and antioxidant enzymes in ammonia intoxication in rats. Free Radic Res 1997; 27: 637-44.

42. Kosenko E, Venediktova N, Kaminsky Y, Montoliu C, Felipo V. Sources of oxygen radicals in brain in acute ammonia intoxication in vivo. Brain Res 2003; 981: 193-200.

43. Görg B, Qvartskhava N, Keitel V, et al. Ammonia induces RNA oxidation in cultured astrocytes and brain in vivo. Hepatology 2008; 48: 567-79.

44. Mehrotra A, Trigun SK. Moderate grade hyperammonemia induced concordant activation of antioxidant enzymes is associated with prevention of oxidative stress in the brain slices. Neurochem Res 2012; 37: 171-81.

45. Schliess F, Görg B, Fischer R, et al. Ammonia induces MK-801-sensitive nitration and phosphorylation of protein tyrosine residues in rat astrocytes. FASEB J 2002; 16: 739-41.

46. Singh S, Koiri RK, Trigun SK. Acute and chronic hyperammonemia modulate antioxidant enzymes differently in cerebral cortex and cerebellum. Neurochem Res 2008; 33: 103-13.

47. Jiang W, Desjardins P, Butterworth RF. Hypothermia attenuates oxidative/nitrosative stress, encephalopathy and brain edema in acute (ischemic) liver failure. Neurochem Int 2009; 55: 124-8.

48. Görg B, Qvartskhava N, Bidmon H-J, et al. Oxidative stress markers in the brain of patients with cirrhosis and hepatic encephalopathy. Hepatology 2010; 52: 256-65.

49. Pun PBL, Lu J, Moochhala S. Involvement of ROS in BBB dysfunction. Free Radic Res 2009; 43: $348-64$.

50. Butterworth RF. The liver-brain axis in liver failure: neuroinflammation and encephalopathy. Nat Rev Gastroenterol Hepatol 2013; 10: 522-8.

51. Cauli O, Rodrigo R, Piedrafita B, Boix J, Felipo V. Inflammation and hepatic encephalopathy: ibuprofen restores learning ability in rats with portacaval shunts. Hepatology 2007; 46: 514-9.

52. Perazzo JC. Hepatic encephalopathy: an approach to its multiple pathophysiological features. World J Hepatol $2012 ; 4: 50$. 